\title{
Acesso a coleções de dados usando LINQ
}

\section{Resumo}

O LINQ (Language INtegrated Query) é um conjunto de extensões introduzido pela Microsoft no .NET Framework 3.5 e nas novas versões da linguagens Visual C\# e Visual Basic que tem como objetivo unificar o modelo de acesso à dados armazenados na memória principal de um computador ou em um repositório de dados externo como um banco de dados ou arquivos XML. Sua sintaxe é equivalente à sintaxe da linguagem SQL usada na comunicação com um sistema gerenciador de banco de dados.Usando o LINQ não é necessário escrever comandos expressos na linguagem SQL embutidos no código de acesso à dados escrito em Visual C\# ou Visual Basic, isto é, pode-se escrever o código de acesso a dados diretamente em Visual C\# ou Visual Basic. Além disso, os dados não precisam estar armazenados somente em um banco de dados. O processamento de uma coleção de dados exige o uso de estruturas de repetição e de comparação para separar os itens que atendam a uma determinada condição de seleção. Este tipo de programação é chamado de programação imperativa porque especifica as ações que devem ser executadas para produzir determinado resultado. Usando LINQ o programador deve especificar somente as condições que os itens de determinada coleção devem atender para serem selecionados, isto é, usa-se a programação declarativa. Neste tipo de programação não se especifica como obter o resultado o compilador $\mathrm{C \#}$ gera todo o código que implementa a declaração automaticamente diminuindo sensivelmente a complexidade do código escrito pelo programador.

\section{Abstract}

The LINQ (Language INtegrated Query) is a set of extensions introduced by Microsoft in the. NET Framework 3.5 and new versions of the language Visual C\# and Visual Basic that aims to unify the model of access to data stored in main memory of a computer or in an external data repository as a database or $X M L$ files. Its syntax is equivalent to the syntax of the SQL language used in communication with a database management system. Using LINQ is not necessary to write $S Q L$ commands expressed in the language embedded in the data access code written in Visual C\# or Visual Basic, that is, you can write code to access data directly in Visual $C$ \# or Visual Basic. Furthermore, the data need not be only stored in a data base. The processing of a collection of data requires the use of repetition and comparison structures to separate the items that meet a certain condition of selection. This type of programming is called imperative programming because it specifies the actions that must be performed to produce a certain result. Using LINQ the programmer must specify only the conditions that the items given collection must meet to be selected, that is, he uses a declarative programming. In this type of programming you didn't specify how

\footnotetext{
1 João Alexandre Magri. Mestre em Engenharia. Professor e Coordenador do curso de Sistemas de Informação da FICS onde, desde o primeiro semestre de 2012, compõe a Gupo de Pesquisa - Oficina de Artigos e Inicialção Científica das Faculdades Integradas Campos Salles - FICS. www.campossalles.edu.br
} 
to obtain the result: the $C$ \# compiler generates all the code that implements the statement automatically reducing substantially the complexity of the code written by the programmer.

\section{Apresentação}

Determinadas aplicações exigem que os dados armazenados nos objetos sejam armazenados na memória auxiliar visando a sua reutilização. Neste caso diz-se que os dados devem ser persistidos e os objetos onde eles estão armazenados são chamados de objetos persistentes.

Em geral os dados são armazenados em arquivos ou em um banco de dados. Nas aplicações de sistemas de informação; por exemplo, é necessário armazenar os dados de um cliente, funcionário, produto, etc. para a sua utilização posterior.

No caso das aplicações desenvolvidas com o paradigma de orientação a objetos, pode-se usar os sistemas gerenciadores de banco de dados orientados a objetos na implementação dos objetos persistentes, principalmente aqueles que representam dados complexos tais como as como figuras, entretanto esta tecnologia ainda encontra-se em processo de desenvolvimento sendo pouco utilizada.

Atualmente a tecnologia padrão corresponde aos sistemas gerenciadores de dados que usam o modelo relacional onde os dados são armazenados em tabelas. Versões mais recentes de alguns destes sistemas gerenciadores de banco de dados relacionais dispõem de extensões orientadas a objetos, portanto tem-se uma tecnologia híbrida chamada de objeto-relacional.

A maior desvantagem dos sistemas gerenciadores de banco de dados objetorelacionais é a total dependência do fabricante o que certamente não é uma boa solução.
Nestas condições, os sistemas gerenciadores de banco de dados são usados para o armazenamento dos objetos persistentes usando-se um mecanismo de montagem e desmontagem destes objetos. A desmontagem permite o armazenamento dos dados contidos em um objeto persistente em determinada linha de uma tabela do modelo relacional. No caso da montagem, usa-se a operação inversa.

As diferenças entre os paradigmas: relacional e orientado a objetos acarretam várias dificuldades que são chamadas de problemas de impedância, sendo que em determinadas situações é necessário usar um programa para fazer o mapeamento de um objeto persistente para uma linha de uma tabela, isto é, fazer o mapeamento objetorelacional. Estes programas são chamados de ferramenta de O/RM (Object / Relational Mapping).

No processo de mapeamento as classes são representadas por tabelas do banco de dados e os objetos persistentes por linhas da tabela correspondente a uma determinada classe. Um dos programas projetados para realizar este mapeamento $\mathrm{O} / \mathrm{R}$ é o Hibernate, desenvolvido para a plataforma Java (NHibernate para plataforma .NET).

Usando-se estas ferramentas não é necessário escrever comandos SQL para acessar os dados do banco de dados porque os comandos são gerados em tempo de execução pelo próprio NHibernate. Além disso, ele suporta os principais sistemas gerenciadores de banco de dados disponíveis no mercado, facilitando o desenvolvimento multiplataforma.

Com o objetivo de minimizar estes problemas de impedância a Microsoft criou uma camada de software intermediária chamada Entity Framework (EF) que permite converter os objetos da camada de negócios da aplicação em linhas das tabelas de um banco de dados relacional e vice versa. 
Ao garantir esta camada de abstração o EF permite que o desenvolvedor se concentre na camada de negócios da aplicação simplificando sensivelmente o trabalho de implementação de um sistema.

O EF possui três opções de consultas:

. Entity SQL (ESQL);

. LINQ to Entities;

. Entity Client;

O LINQ foi criado originalmente para consultar objetos armazenados em memória principal. Depois foi estendido com implementações específicas para poder acessar dados armazenados em qualquer tipo de memória. Assim tem-se as seguintes encarnações do LINQ:
LINQ para objetos
.LINQ para coleções
LINQ para SQL
.LINQ para entidades
.LINQ para XML

$\mathrm{Na}$ figura 1.1 tem-se a arquitetura geral do LINQ.

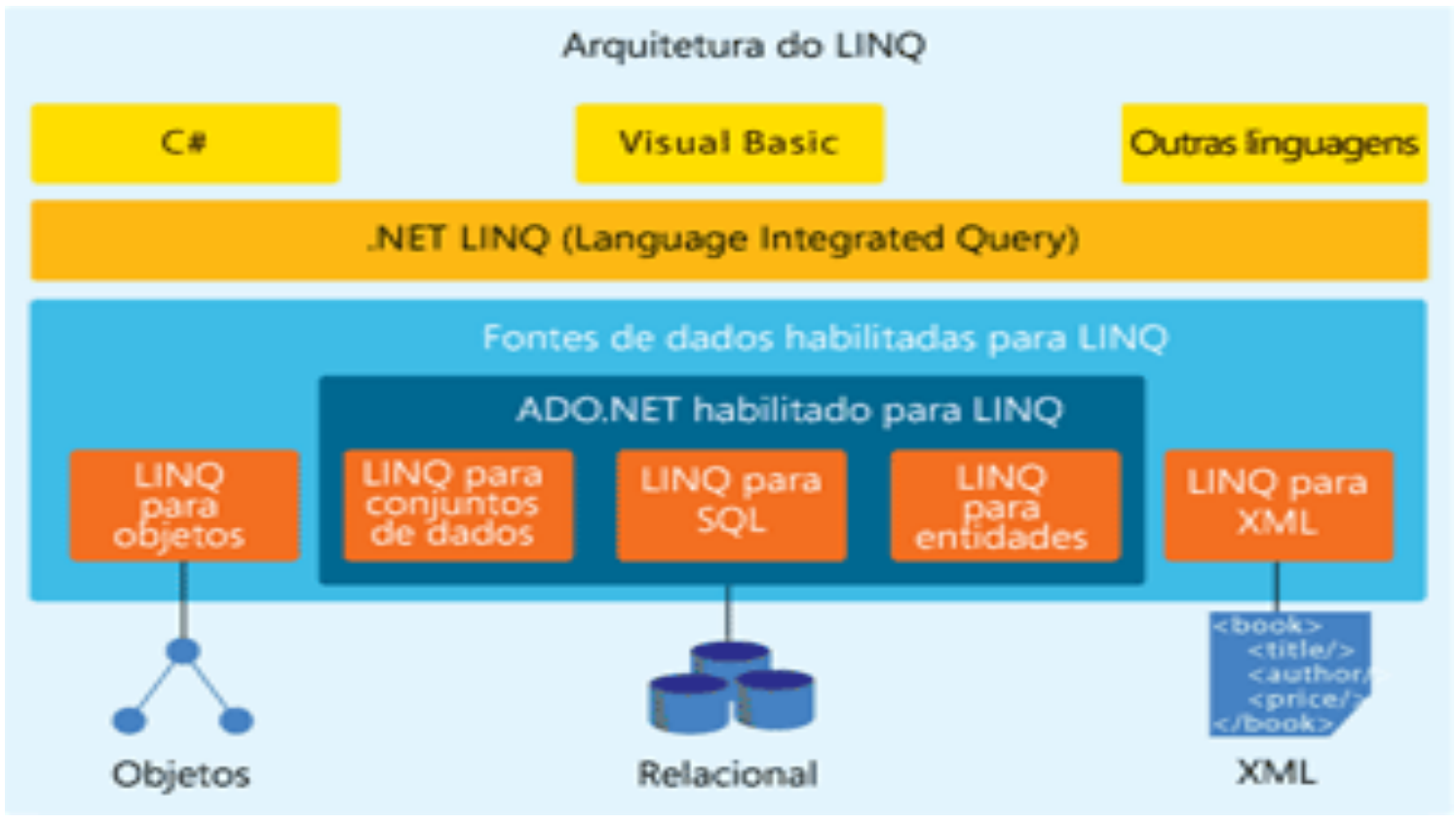

Figura 1.1 Arquitetura geral do LINQ

Fonte: http://msdn.microsoft.com/msdnmag/issues/07/06/CSharp30/pt/fig01.gif

\section{Ferramenta O/R Designer}

No caso das consultas aplicadas a um conjunto de dados armazenados em disco cujo acesso é controlado pelo sistema gerenciador de banco de dados SQL Server deve-se usar a encarnação do LINQ chamada LINQ to SQL.

O mapeamento das tabelas do banco de dados para classes de negócio do sistema pode ser feito usando-se a ferramenta $O / R$ Designer que é fornecida gratuitamente pela
Microsoft no Visual Studio 2008 e portanto também está disponível nas edições Express das linguagens visual $\mathrm{C} \#$ e visual Basic.

Nestas condições, em nível de desenvolvimento do sistema usando metodologia orientada a objetos, deve-se entre outras coisas, desenvolver o diagrama de classes e depois mapeá-lo para o modelo lógico do banco de dados.

O mapeamento das tabelas do banco de dados para as classes de negócio da aplicação é feito usando-se a ferramenta O/R Designer: 
neste processo é gerada automaticamente a classe Datacontext: esta classe contém os métodos para acessar as tabelas do banco de dados, isto é, o código necessário para a implementação das operações CRUD (cadastrar, consultar, atualizar, excluir) sobre os dados armazenados nas tabelas do banco de dados. Quando o banco de dados tiver stored procedures elas serão mapeadas como métodos da classe DataContext. O código de acesso a dados é gerado considerando-se o uso do acesso desconectado que tem grandes vantagens em relação ao acesso conectado quando o volume de conexões é alto, isto é, o acesso conectado está sendo abandonado nos produtos da Microsoft.

Nestas condições, os comandos LINQ serão aplicados aos objetos instanciados a partir da classe DataContext. Pode-se gerar uma classe DataContext para todo o banco de dados ou somente para algumas tabelas do banco, isto é, em um projeto pode-se ter várias classes DataContext portanto é corrente usar o nome xxxDataContext onde xxx identifica o seu conteúdo. Quando for gerada uma classe DataContext para todas as tabelas do banco de dados, xxx será o nome do banco de dados.

A grande vantagem da ferramenta $\mathrm{O} / \mathrm{R}$ Designer é o recurso de geração das operações CRUD automaticamente, isto é, o programador não precisa codificar a camada de acesso a dados do banco de dados, devendo se preocupar somente com o desenvolvimento das classes de negócio da aplicação o que aumenta sensivelmente a sua produtividade.

3. Montage m d a c las s e xxxDataContext

$\mathrm{Na}$ figura 3.1 tem-se as tabelas de um banco de dados chamado Vendas Geral composto pelas tabelas Cliente e Pedido.

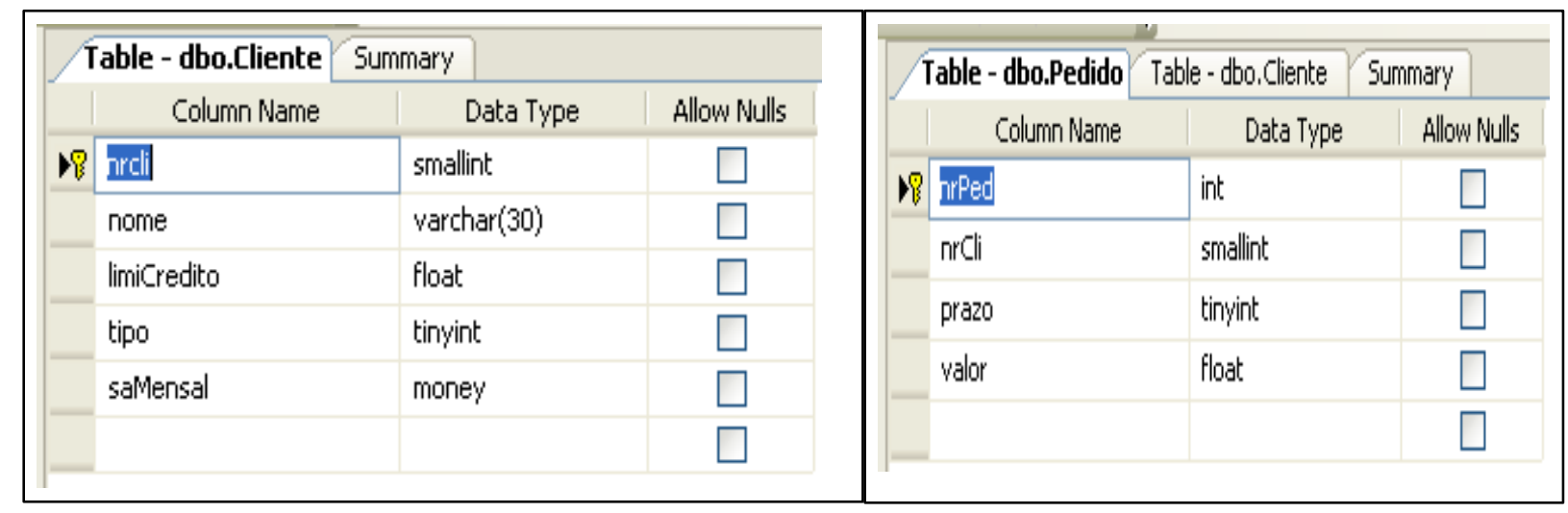

Figura 3.1 - Tabelas que compõem o banco de dados Vendas Geral

Utilizando-se a ferramenta O/R Designer estas tabelas foram mapeadas para as classes de negócio cliente e Pedido, conforme pode ser visto na figura 3.2 


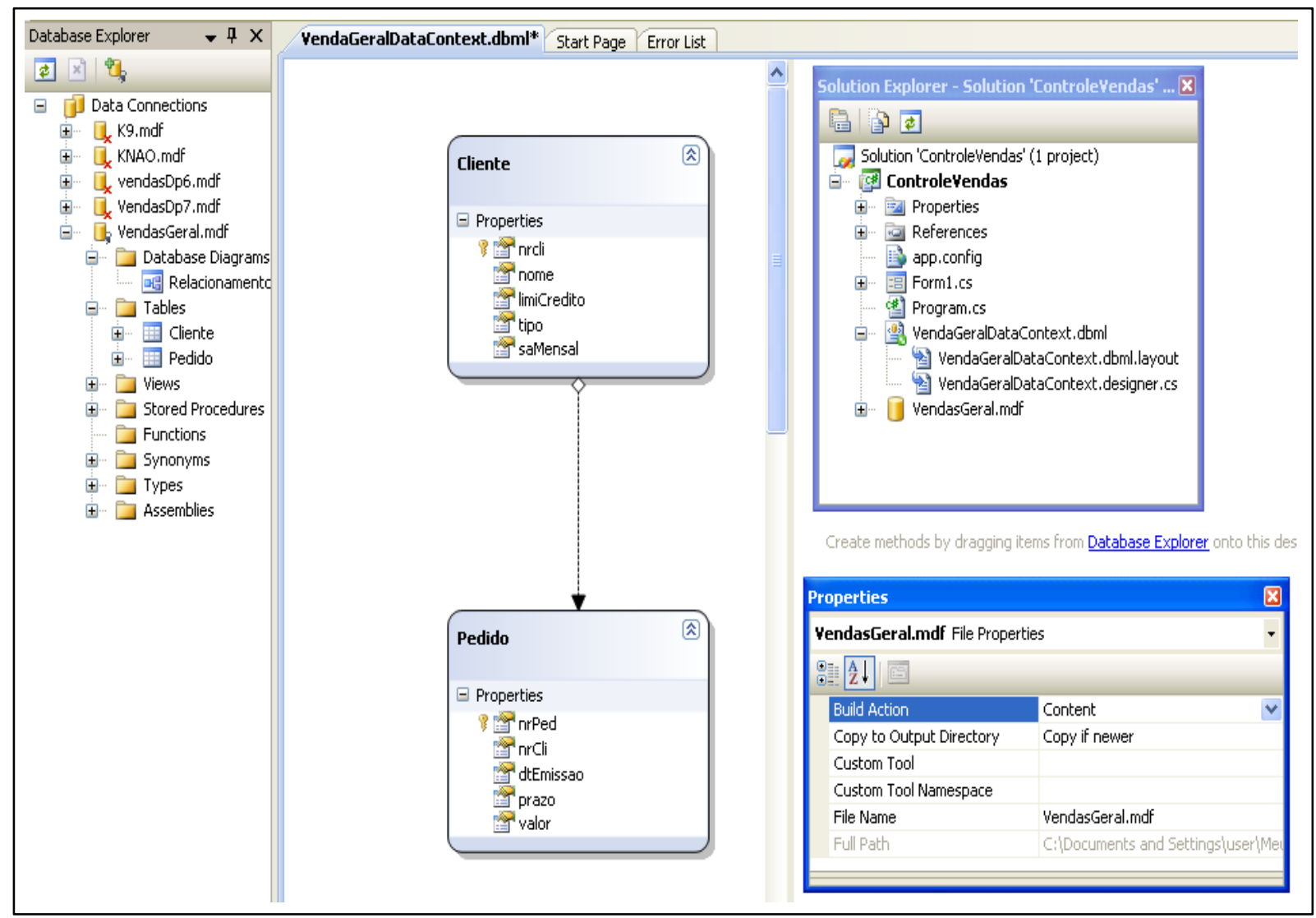

Figura 3.2 - Diagrama de classes do sistema gerado com a ferramenta O/R Designer

Observar na janela Solution Explorer que foi criada a classe Venda Geral Data Context que contem todo o código de acesso a dados do banco de dados gerado automaticamente pela ferramenta $\mathrm{O} / \mathrm{R}$ Designer.

\section{Listagem de um conjunto de linhas de uma tabela usando LINQ}

Nos exemplos a seguir serão feitas consultas às tabelas Cliente e Pedido do banco de dados Vendas Geral. Elas serão apresentadas em duas versões:
I. Versão expressa na linguagem SQL;

II. Versão expressa na linguagem LINQ;

É importante observar que no caso da versão LINQ Cliente e Pedido são os nomes das classes cujo código foi gerado automaticamente pela ferramenta $O / R$ Designer: as tabelas do banco de dados foram chamadas de Clientes e Pedidos, isto é, o O/R Designer acrescentou automa-ticamente a letras no final dos nomes das classes para nomear as tabelas do banco de dados.

Exemplo 1 - Emitir a relação de clientes da empresa 


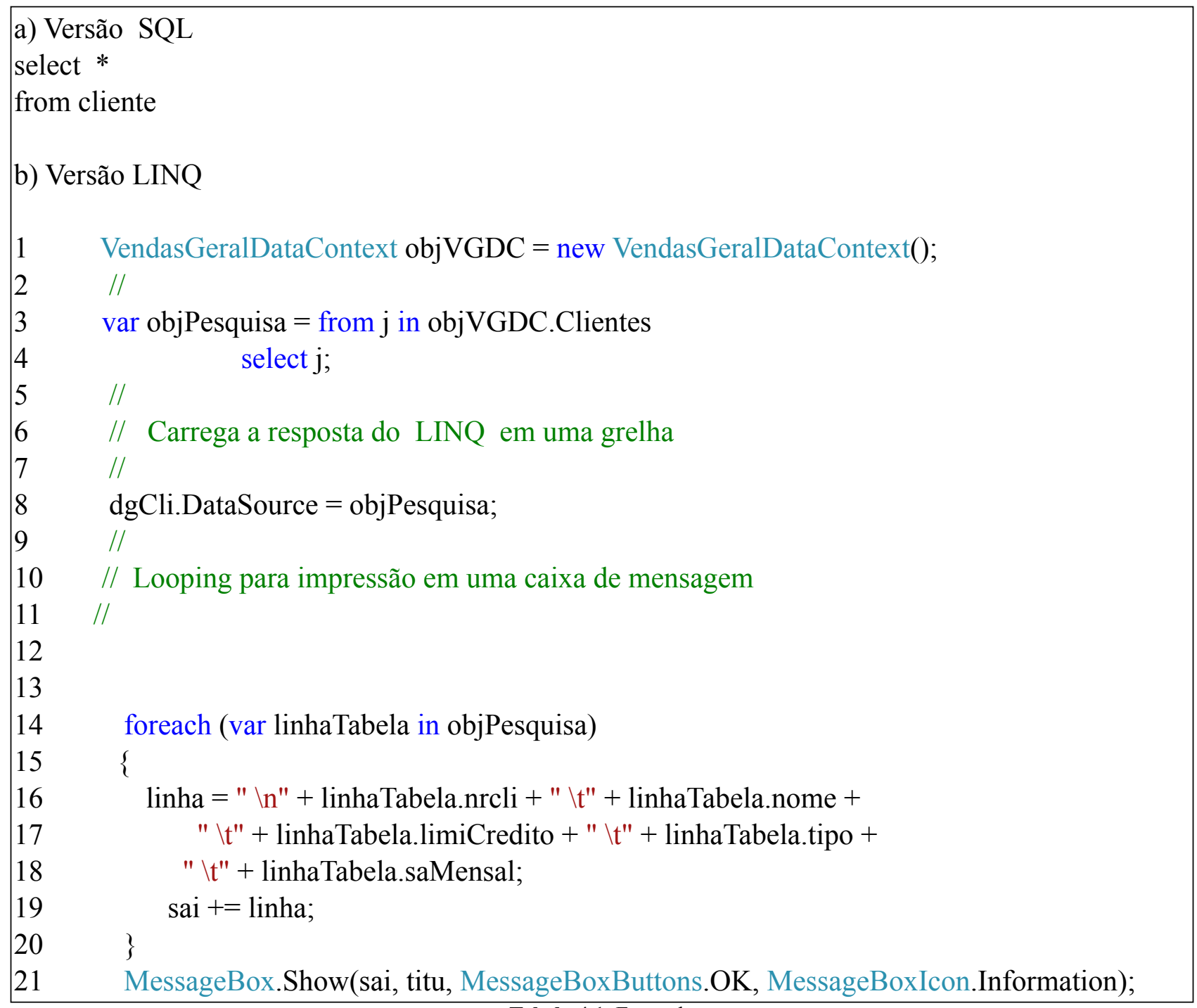

Tabela 4.1. Exemplo

Ao ser executado este comando LINQ obtém-se a resposta apresentada na Figura 3.3

Deve-se observar que antes de ser executado o comando LINQ escrito nas linhas 3 e 4 foi instanciado um objeto da classe ponte Vendas Geral Data Context na linha número 1. Este objeto contém todos os métodos de acesso ao banco de dados Vendas Geral. Conforme já foi explicado, os comandos LINQ são executados sobre as tabelas do projeto da aplicação e não sobre o banco de dados físico. Assim na linha número 3 foi acessada a tabela Clientes cujo nome está escrito no plural.

$O$ resultado da execução de um comando LINQ é um conjunto de objetos anônimos: eles passam a ser a fonte de dados para o objeto de tela ou controle Data Grid: na linha número 8 este conjunto de objetos foi vinculado ao objeto de tela visando apresentar o resultado do processamento em uma grelha conforme pode ser visto na Figura 3.3.

Opcionalmente pode-se processar cada um dos objetos do conjunto de objetos resposta: nas linhas de 14 a 21 os valores das variáveis de instância dos objetos foram impressos em uma caixa de mensagem conforme pode ser visto na Figura 3.3. Neste caso foi montado um looping usando-se a estrutura de repetição foreach, aberta na linha número 14. A variável de controle da estrutura de repetição linha Tabela representa cada uma das variáveis de referência do conjunto de objetos resposta, portanto, para acessar determinado 
valor de umas das variáveis de instância destes objetos foi usada a sintaxe linha Tabela. Nome da variável de instância conforme está mostrado nas linhas números 16 a 18 .

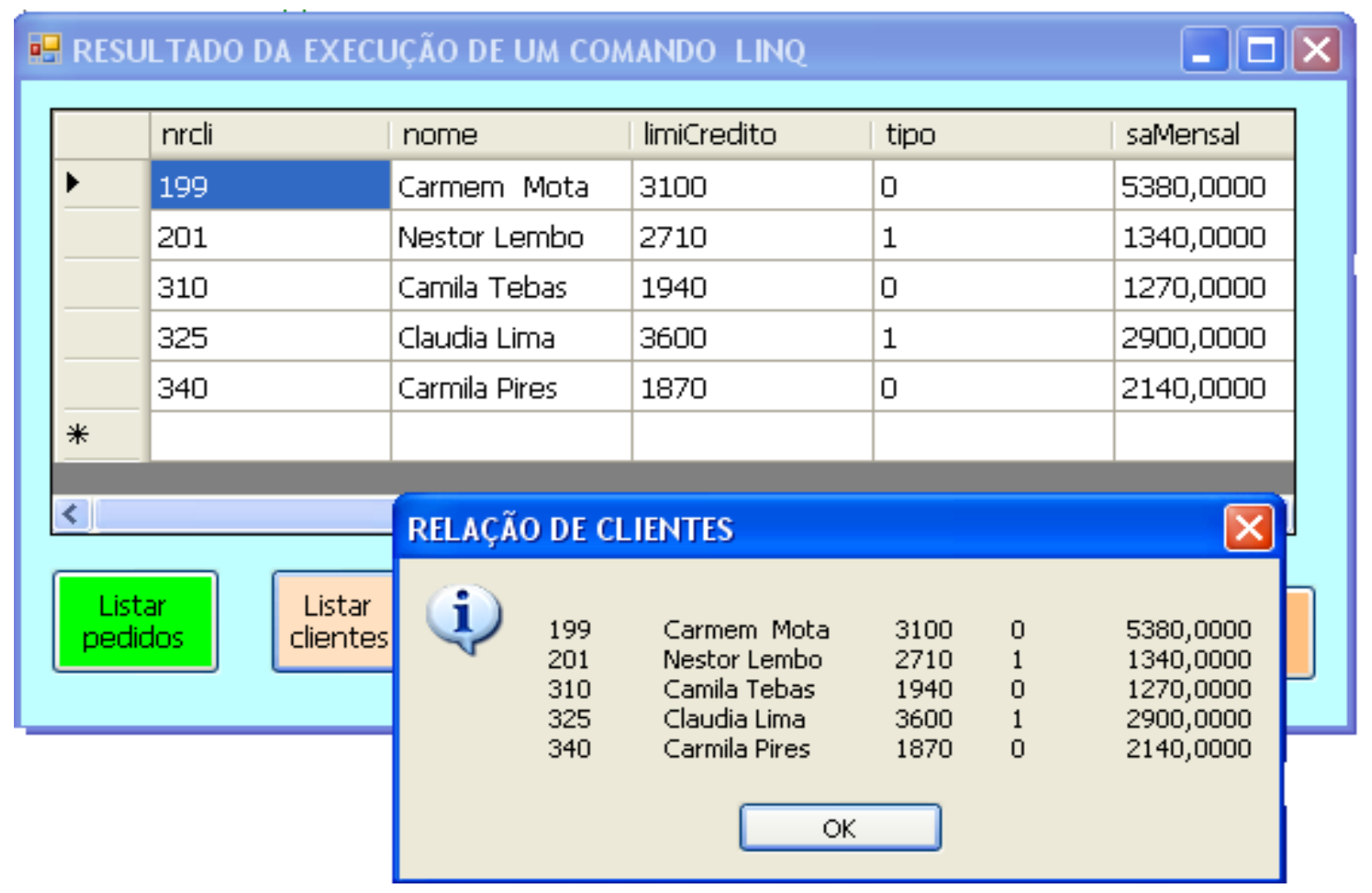

Figura 3.3 - Resultado da execução do comando LINQ para listar uma tabela.

Exemplo 2 - Listar os dados dos clientes cujo limite de crédito é maior do que $\mathrm{R}$ $\$ 1340,00$ e o salário mensal maior do que $\mathrm{R}$
$\$ 1800,00$. A listagem deverá ser ordenada pelos nomes dos funcionários.

\section{a)Versão SQL \\ select $*$ \\ from cliente}

b) Versão LINQ

where limiCredito $>1340$ and saMensal $>1800$

order by nome

1 VendasGeralDataContext objVGDC = new VendasGeralDataContext();

$2 \quad / /$

3 var objPesquisa $=$ from $r$ in objVGDC.Clientes

$4 \quad$ where r.limiCredito $>1340 \& \&$ r.saMensal $>1800$

$5 \quad$ orderby r.nome

$6 \quad$ select $\mathrm{r}$;

$7 \quad / 1$

$8 \quad$ // Carrega a resposta do LINQ em uma grelha 


\begin{tabular}{|c|c|c|c|c|c|c|}
\hline 9 & // & & & & & \\
\hline 10 & dgCli.DataSource = objPesquisa; & & & & & \\
\hline 11 & $/ /$ & & & & & \\
\hline 12 & // Looping para impressão em uma caixa d & 1 & isagem & & & \\
\hline 13 & $/ /$ & & & & & \\
\hline 14 & foreach (var linhaTabela in objPesquisa) & & & & & \\
\hline 15 & \{ & & & & & \\
\hline 16 & linha $=" \backslash n "+$ linhaTabela.nrcli $+" \backslash t^{\prime}+$ & 1 & aTabela.no & ne + & & \\
\hline 17 & $" \backslash \mathrm{t} "+$ linhaTabela.limiCredito $+" \backslash \mathrm{t} "$ & + & haTabela.ti & po + & & \\
\hline 18 & " \t" + linhaTabela.saMensal; & & & & & \\
\hline 19 & sai += linha; & & & & & \\
\hline 20 & \} & & & & & \\
\hline 21 & MessageBox.Show(sai, titu, MessageBoxE & I & $\begin{array}{l}\text { NroPedido } \\
2120\end{array}$ & $\begin{array}{l}\text { Valor } \\
380,51\end{array}$ & Prazo & \begin{tabular}{|l|} 
Posicao \\
Em processamento
\end{tabular} \\
\hline & Tabela 3.2 & & 2310 & 420,54 & 2 & Pedido entregue \\
\hline & & & $\frac{2315}{2340}$ & $\begin{array}{l}910 \\
350,42\end{array}$ & $\begin{array}{l}3 \\
6\end{array}$ & $\begin{array}{l}\text { Em processamento } \\
\text { Em processamento }\end{array}$ \\
\hline & ser executado este comand & & 2345 & 510,85 & 2 & Pedido entregue \\
\hline & ser execulado este & & 2440 & 240,87 & 2 & Pedido entregue \\
\hline obt & -se a resposta apresentada na Figura 3.4. & & 2445 & 960,37 & 3 & Em processamento \\
\hline
\end{tabular}

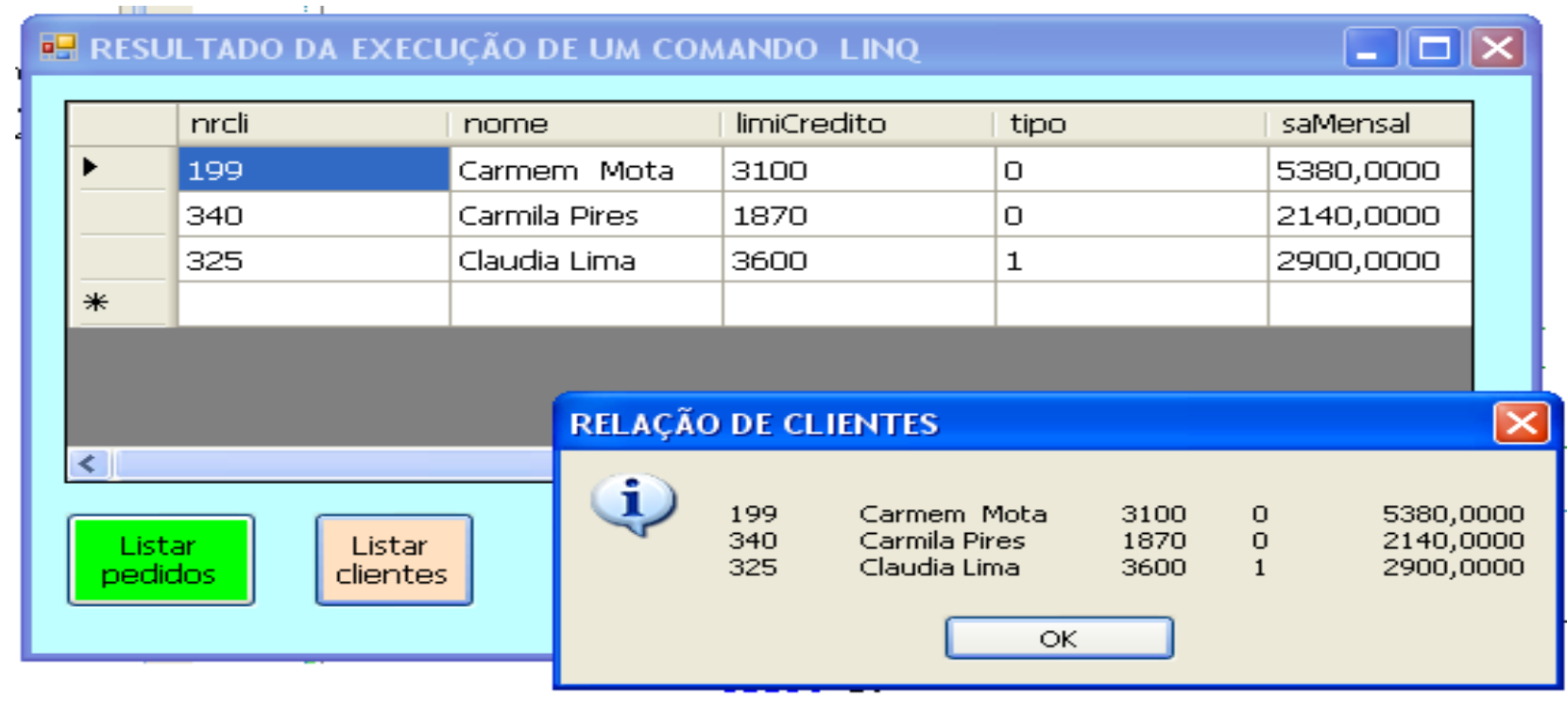

Figura 3.4 - Resultado da execução do comando LINQ para listar parte de uma tabela.

Observar que o comando LINQ está escrito nas linhas números 3 a 6 : as outras linhas correspondem à vinculação do conjunto de objetos resposta a uma grelha de apresentação de resultados (linha número 10) e ao processamento de cada um dos objetos do conjunto de objetos resposta: neste caso, os valores de suas variáveis de instância foram impressos em uma caixa de impressão (linhas números 14 a 21).

Exemplo 3: - Emitir a listagem dos pedidos (número do pedido, valor e prazo de entrega). Criar mais uma coluna a ser preenchida usando-se a seguinte regra do negócio: se o prazo for menor do que três dias esta coluna deverá ter o valor "Pedido entregue". Se não for deverá ter o valor "Em processamento". Os nomes das colunas da grelha de apresentação deverão ser: Nro Pedido, Valor, Prazo e Posição e não aqueles adotados para as colunas da tabela Pedido.

O comando LINQ para a execução desta consulta e a respectiva resposta estão apresentados na figura 3.6. 


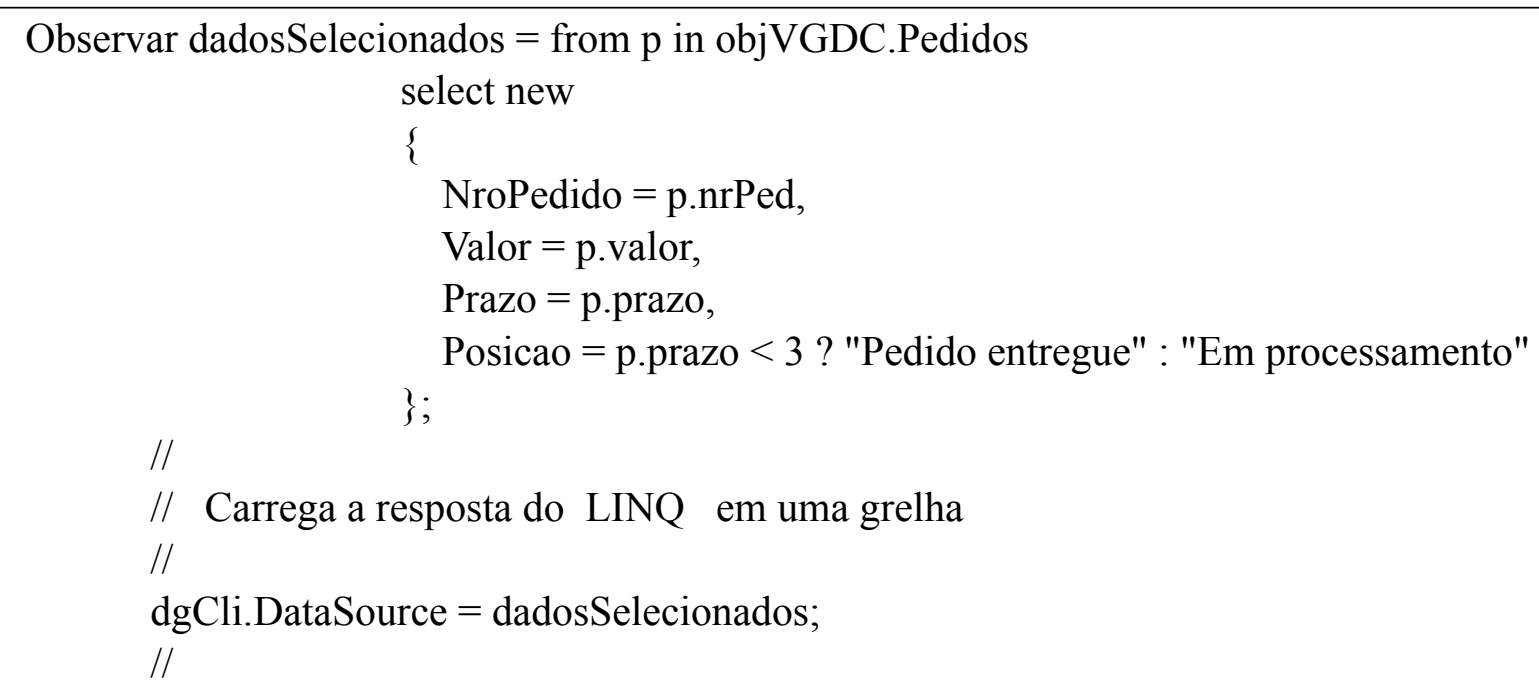

Figura 3.6 - Comando LINQ para alterar os nomes das colunas da grelha e criar uma nova coluna.

Para alterar os nomes das variáveis de instância do conjunto de objetos resposta foi criada uma classe anônima e instanciado um objeto desta classe. As variáveis de instância destes objetos foram iniciadas com os valores das variáveis de instância do conjunto de objetos resposta.

A nova coluna chamada Posição também é uma variável de instância da classe anônima: sua variável de instância é iniciada usando-se a estrutura de comparação iif, conforme pode ser visto na figura 3.6.

\section{Considerações finais}

A sintaxe da linguagem LINQ é muito parecida com a sintaxe da linguagem SQL, porém mais eficiente e explora o conceito de objetos na composição dos comandos. Além disso, ela é uma extensão das linguagens visual $\mathrm{C \#}$ e visual Basic e não uma nova linguagem a ser embutida no interior de um programa escrito em C\#, com sintaxe própria.
Os comandos LINQ são executados sobre um objeto instanciado a partir da classe Data context que tem os métodos para executar as operações CRUD automaticamente gerados pelo Visual Studio, isto é, o programador não precisa codificar a camada de acesso da aplicação que é uma das mais extensas e repetitivas. Ele precisa codificar apenas as classes de negócio da aplicação o que representa um ganho significativo em termos de produtividade do programador.

Muitos profissionais têm dúvidas a respeito da geração automática de modelos e de parte do código, afirmando que os resultados não são eficientes. Entretanto, eles não avaliam o resultado da tradução de um código fonte para linguagem de máquina ou bytescodes gerados pela "compilação" de um código escrito em Java. Basicamente programa executável é gerado a partir do código fonte.

No ambiente Microsoft está se observando um amadurecimento no processo de desenvolvimento de software.

O ganho em termos de tempo de desenvolvimento é substancial. 
O Framework .NET 3.5 possui a maioria das rotinas que os programadores eram obrigados a codificar com freqüência, evitando o clássico retrabalho. Além disso, ferramentas com o visual Studio preocupamse em gerar, de forma automática, todo o código que não tem a ver com o desenvolvimento da aplicação em si.

Nessas condições, a geração de código e modelos é uma tecnologia que está sendo adotada por todas as empresas que desenvolvem software.

\section{Referências Bibliográficas}

MAGRI, J. A. Programação C\#. 1. Ed. São Paulo: Editora Érica, 2012.

MAGRI, J. A . e RODRIGUES, V. B. Princípios da Arquitetura Dirigida a Modelos (MDA).

Trabalho de Conclusão de Curso (Graduação em Ciência da Computação). Fundação Instituto Tecnológico de Osasco, São Paulo, 2007.

MARGARIE, F. LINQ in action. 1. ed. California: O’ reilly Media Inc, 2008.

NAGEL, C. Professional C\# and .NET 4. 1. ed. Indianapolis: Wiley Publishing, Inc, 2010

PIARLOSI, P. Programming Microsoft LINQ in Microsoft .NET framework 4.0. 1. ed. California: O’ reilly Media Inc, 2010.

RATTZ, J. Pro LINQ: Integrated Query in C\# 2010. 1 ed. Indianapolis: Wiley Publishing, Inc, 2010.

SHARP, J. Microsoft Visual C\# 2008. 1. ed. Washington: Microsoft Press, 2008.

STELLMAN, A. Head First C\#. 1. ed. California: O’ reilly Media Inc, 2008. 